・研究报告・

\title{
我国网络平台外来宠物贸易调查
}

\author{
姬云瑞 ${ }^{1,2}$ 李 叶1,2 刘 芳1,2 李迪强1,2* \\ 1 (中国林业科学研究院森林生态环境与保护研究所, 北京 100091) \\ 2 (国家林业和草原局生物多样性保护重点实验室, 北京 100091)
}

\begin{abstract}
摘要: 为了解我国网络平台外来宠物贸易情况，作者分别在2018年、2019年对爬宠网app、转转app、微信和慧聪 网 4 个网络平台上外来宠物贸易的种类、规模、价格进行了为期各 6 周的调查。共记录到外来宠物 111 种, 隶属于 4 纲 8 目 44 科 94 属, 其中爬行纲 3 目 31 科 78 属 94 种, 占物种总数的 $84 \%$; 两栖纲 2 目 9 科 11 属 12 种, 占物种总数的 $11 \%$; 鸟纲 1 目 2 科 3 属 3 种, 占物种总数的 $3 \%$; 哺乳纲 2 目 2 科 2 属 2 种, 占物种总数的 $2 \%$ IUCN红色名录受威胁物种 27 种, 占物种总数的 $24 \%$; 列入CITES附录的物种 45 种, 占物种总数的 $41 \%$ 。 $77 \%$ 的外来贸易宠物售卖信息条数低于6条, $58 \%$ 的外来贸易宠物价格低于 1,000 元/只。本文根据目前网络平台外来宠物贸易现状, 提出了网络平台动物贸易监 管建议。
\end{abstract}

关键词: 外来宠物; 动物贸易; 网络平台; 管理措施

\section{Assessment of current trade of exotic pets on the internet in China}

\author{
Yunrui $\mathrm{Ji}^{1,2}$, Ye $\mathrm{Li}^{1,2}$, Fang $\mathrm{Liu}^{1,2}$, Diqiang $\mathrm{Li}^{1,2^{*}}$ \\ 1 Institute of Forest Ecology, Environment and Protection, Chinese Academy of Forestry, Beijing 100091 \\ 2 Key Laboratory of Biodiversity Conservation, State Forestry and Grassland Administration, Beijing 100091
}

\begin{abstract}
In order to investigate the trade of exotic pets in China, two 6-week surveys were conducted separately in 2018 and 2019 to analyze the type, volume, and price for sale of exotic pets on four online platforms (Pachongwang app, Zhuanzhuan app, Wechat and Huicongwang). A total of 111 exotic species were recorded across 4 classes, 8 orders, 44 families, and 94 genera-including 94 species $(84 \%, n=111)$ in Reptilia, 12 species (11\%) in Amphibia, 3 species (3\%) in Aves, and 2 species (2\%) in Mammalia. Twenty-seven species of exotic pets (24\%) were listed as threatened species by IUCN Red List and 45 species (41\%) were listed in the CITES Appendix. The number of trade events for $77 \%$ of species were fewer than six during the survey. Prices of 58\% of species were lower than 1,000 yuan per individual. We propose reforms to the current regulation of the online animal and exotic pet trade given the concerning findings of this study.
\end{abstract}

Key words: exotic pets; animal trade; Internet; management measures

自古以来, 宠物就是人类文化的一部分(Bush et al, 2014)。随着全球经济的发展和人群购买力的 提升, 人们对宠物的选择越来越多样化和个性化, 越来越多的外来动物进入宠物市场(梦梦等, 2017)。 宠物需求成了全球动物贸易的重要驱动力之一 (Moorhouse et al, 2017)。大量的外来物种贸易不仅 会加剧濒危物种的灭绝(Annorbah et al, 2016; Shepherd et al, 2016), 还会导致外来物种入侵(Carrete
\& Tella, 2008; Su et al, 2016; Lockwood, 2017)和世 界性的传染病传播(Harkins et al, 2014)等问题。

随着互联网的快速发展及其便捷性的提高, 互 联网平台已经成为动物贸易的重要平台。国际爱护 动物基金会(IFAW) 2005年发布的动物网络贸易报 告中指出, eBay等在线贸易网站中存在鹦鹉、猛禽 等野生动物的贸易(Siriwat \& Nijman, 2018)。近几 年, 动物网络贸易还从传统的电子商务平台 (eBay、

收稿日期: 2019-12-07; 接受日期: 2020-05-24

基金项目: 中国林业科学研究院科研机构基本业务费(169160004000180004)

* 通讯作者 Author for correspondence. E-mail: lidiq@sina.com 
Amazon等)扩展到社交媒体平台(Instagram、Facebook等)(Siriwat et al, 2019)。国内调查发现百度贴 吧、微信、快手等网络平台存在野生动物贸易(马波 等, 2013)。频繁的外来物种网络贸易会加剧入侵物种 的传播，挑战生物安全(Derraik \& Phillips, 2010)。网 络平台上的动物贸易隐蔽性高, 增加了监管工作难 度(Sung \& Fong, 2018)。为加强网络动物贸易监管, 就需全面了解网络动物贸易的物种和规模。

国外关于网络平台上动物贸易的研究较多, 包 括哺乳类(Harrison et al, 2016; Siriwat \& Nijman, 2018)、爬行类(Jensen et al, 2019)、鸟类(Martin et al, 2018)。我国关于动物贸易的研究主要集中在实体市 场调查(Cheung \& Dudgeon, 2006; Gong et al, 2006, 2009; 梦梦等, 2017; 王健等, 2018)、进出口海关和 跨境口岸调查(熊小倩等, 2017; 梦梦等, 2018)等, 而网络平台上的动物贸易调查较少, 主要进行了龟 类和鹦鹉类的调查(Sung \& Fong, 2018; Ye et al, 2020), 缺少其他类群动物的贸易情况。为此, 本文 对网络平台上外来宠物贸易的种类、规模、价格进 行调查和分析, 以期为制定更加合理有效的外来物 种网络贸易监管办法提供科学依据。

\section{调查方法}

选择4个有代表性的网络平台, 即爬宠网app、 转转app、微信(公众号)和慧聪网(https://www.hc360. $\mathrm{com} /$ )。对这几个平台进行 2 次各为期6周的调查, 调 查时间为2018年9-11月和2019年3-5月。调查对象 为网络平台上所售卖的外来宠物, 主要有两栖类、 爬行类、鸟类和哺乳类。调查方法为汶览网络平台, 篮选出涉及外来宠物的售卖信息，尽可能记录下发 布时间、物种名称和价格。物种确认是基于网络平 台中提供的学名或俗名并结合图片信息, 若遇到无
法确定的物种，将卖家提供的图像与图鉴资料进行 比对，或咨询相关专业人士。为了避免重复记录， 调查过程中记录售卖信息发布者的昵称、所在位置 和售卖物种的性别、大小等信息，通过对比具体信 息，剔除掉重复数据。

物种中文名参照《拉汉英两栖爬行动物名称》 (赵尔宓等, 1993)、《中国贸易龟类检索图鉴》(史 海涛, 2008)、《世界哺乳动物名典》 (汪松等, 2001) 和《世界鸟类分类与分布名录》 (郑光美, 2002), 四 书未有收录者则按照学名释译; 物种学名及分类系 统参照Species 2000 \& ITIS Catalogue of Life (Roskov et al, 2019); 保护等级依据《濒危野生动植物种国际 贸易公约》(CITES, 2019), 物种濒危等级依据IUCN 濒危物种红色名录(IUCN, 2017)。

\section{2 结果}

\section{1 外来宠物的物种组成}

本次调查共记录到外来宠物 111 种, 隶属于 4 纲 8目44科94属(附录1), 其中爬行纲3目31科78属94种, 占物种总数的 $84 \%$; 两栖纲 2 目 9 科 11 属 12 种，占物种 总数的 $11 \%$; 鸟纲 1 目 2 科 3 属 3 种，占物种总数的 $3 \%$; 哺乳纲 2 目 2 科 2 属 2 种，占物种总数的 $2 \%$ (表1)。

\section{2 外来宠物贸易的规模}

通过网络平台上的宠物贸易数据无法获取准 确动物数量, 因此本文选择售卖信息的条数来估计 宠物贸易的规模大小，按照数量分为 4 类: $<6$ 条、 6-10条、10-20条、>20条。调查结果显示，售卖信 息条数少于 6 条的共有 86 种，占物种总数的 $77 \%$; 6-10条和10-20条的分别有15种(14\%)和7种(6\%); > 20 条的只有3种(3\%), 分别是䰂蜥(Pogona vitticeps)、 美洲瓺蜥(Iguana iguana)、黑栉尾蚚(Ctenosaura similis) (表2)。

表1 网络交易外来宠物的种类

Table 1 Types of the exotic pets that traded on the internet

\begin{tabular}{lllll}
\hline 纲 Class & 目 Order & 科数 Number of family & 属数 Number of genus & 物种数 Number of species (\%) \\
\hline 爬行纲 Reptilia & 鳄目 Crocodylia & 1 & 1 & $1(1)$ \\
& 龟鳖目 Testudines & 6 & 26 & $29(26)$ \\
& 有鳞目 Squamata & 24 & 51 & $64(57)$ \\
两栖纲 Amphibia & 无尾目 Anura & 8 & 10 & $11(10)$ \\
& 有尾目 Caudata & 1 & 1 & $1(1)$ \\
鸟纲 Aves & 鹦形目 Psittaciformes & 2 & 3 & $3(3)$ \\
哺乳纲 Mammalia & 啮齿目 Rodentia & 1 & 1 & $1(1)$ \\
& 袋鼠目 Diprotodontia & 1 & 1 & $1(1)$ \\
\hline
\end{tabular}


表2 外来宠物网上贸易规模

Table 2 Volumes of exotic pets traded on the internet

\begin{tabular}{lll}
\hline $\begin{array}{l}\text { 售卖信息条数 } \\
\text { Number of trade } \\
\text { events }\end{array}$ & $\begin{array}{l}\text { 物种数 } \\
\text { Number of } \\
\text { species (\%) }\end{array}$ & $\begin{array}{l}\text { CITES附录I和II物种数 Number } \\
\text { of species of CITES Appendix I } \\
\text { and II (\%) }\end{array}$ \\
\hline$<6$ & $86(77)$ & $30(71)$ \\
$6-10$ & $15(14)$ & $7(17)$ \\
$10-20$ & $7(6)$ & $3(7)$ \\
$>20$ & $3(3)$ & $2(5)$ \\
\hline
\end{tabular}

表3 外来宠物网上报价

Table 3 The prices for sale of the exotic pets traded on the internet

\begin{tabular}{lll}
\hline $\begin{array}{l}\text { 价格 } \\
\text { Price (RMB yuan) }\end{array}$ & $\begin{array}{l}\text { 物种数 } \\
\text { Number of } \\
\text { species (\%) }\end{array}$ & $\begin{array}{l}\text { CITES 附录I和II的物种数 } \\
\text { Number of species of CITES } \\
\text { Appendix I and II (\%) }\end{array}$ \\
\hline$<1,000$ & $50(58)$ & $16(38)$ \\
$1,000-5,000$ & $30(35)$ & $12(29)$ \\
$5,000-10,000$ & $4(5)$ & $2(5)$ \\
$>10,000$ & $2(2)$ & $1(2)$ \\
\hline
\end{tabular}

\section{3 外来宠物贸易的价格}

记录到的 111 个物种中, 86 个物种有价格信息， 占总物种数的 $77 \%, 25$ 个物种缺少价格信息。价格低 于 5,000 元的物种有 80 种，占有价格信息物种数的 $93 \%$ 。价格位于 $5,000-10,000$ 元的 4 个物种中, 有 2 个 CITES附录物种, 即五彩金刚鹦武(Ara macao)和巨 蚚(Varanus cumingi); 价格高于 10,000 元的 2 个物种 中, 有 1 个CITES附录物种, 即喉盾陆龟(Astrochelys yniphora) (表3)。

\section{4 外来宠物的濒危等级}

本次调查到的 111 个物种中, 有 27 种被IUCN 红 色名录列为受胁物种, 占物种总数的 $24 \%$ (表 4 )。其 中有7种被列为极危(CR), 分别是暹罗鳄(Crocodylus siamensis)、乌蒂拉栉尾蚚(Ctenosaura bakeri)、安南 摄龟(Mauremys annamensis)、辐纹陆龟(Astrochelys radiata)、喉盾陆龟、缅甸星龟(Geochelone platynota)、 黑凹甲陆龟(Manouria emys); 6种被列为濒危(EN), 分别是非洲灰财鹉(Psittacus erithacus)、危地马拉栉 尾蚚(Ctenosaura palearis)、五棱栉尾蜥(C. quinquecarinata)、蓝岩瓺蜥 (Cyclura lewisi)、点斑水龟 (Clemmys guttata)、木雕水龟(Glyptemys insculpta)。

在本次调查中, 被列入CITES附录的物种共有 45 种，占物种总数的 $41 \%$ (表5)。其中列入附录I的有 10 种, 分别是暹罗鳄、安南摄龟、辐纹陆龟、喉盾 陆龟、缅甸星龟、印度星龟(Geochelone elegans)、
表4 网络交易外来宠物的IUCN濒危物种红色名录级别

Table 4 IUCN Red List of Endangered Species category of the exotic pets traded on the internet

\begin{tabular}{|c|c|c|}
\hline $\begin{array}{l}\text { IUCN红色名录 } \\
\text { IUCN Red List }\end{array}$ & $\begin{array}{l}\text { 物种数 } \\
\text { Number of species }\end{array}$ & $\%$ \\
\hline 极危 Critically Endangered & 7 & 6 \\
\hline 濒危 Endangered & 6 & 5 \\
\hline 易危 Vulnerable & 14 & 13 \\
\hline 近危 Near Threatened & 5 & 5 \\
\hline 无危 Least Concern & 69 & 62 \\
\hline 未评估 Not Evaluated & 10 & 9 \\
\hline 合计 Total & 111 & \\
\hline
\end{tabular}

表5 网络贸易外来宠物的CITE附录级别及其人工繁殖来 源比例

Table 5 The exotic pets traded on the internet that are listed in CITES Appendix and their captive breeding origin

\begin{tabular}{lll}
\hline $\begin{array}{l}\text { CITES 附录 } \\
\text { CITES Appendix }\end{array}$ & $\begin{array}{l}\text { 物种数 } \\
\text { Number of } \\
\text { species (\%) }\end{array}$ & $\begin{array}{l}\text { 圈养繁殖物种数 } \\
\text { Number of species bred } \\
\text { in captivity (\%) }\end{array}$ \\
\hline I & $10(9)$ & $10(100)$ \\
II & $32(29)$ & $12(38)$ \\
III & $3(3)$ & $2(67)$ \\
未列入附录 Not & $66(59)$ & \\
included in appendix & & \\
\hline
\end{tabular}

非洲灰鹦武、蓝岩鬌蜥、五彩金刚鹦鹉、印度泛棱 背龟(Pangshura tecta)。附录II物种有32种，包括胫 刺陆龟(Centrochelys sulcata)、豹纹陆龟(Stigmochelys pardalis)、球蟒(Python regius)、美洲镾蜊等。本 次调查到的45种CITES附录物种中, 24种(53\%)有圈 养繁殖成功的案例(附录1)。

\section{3 讨论}

\section{1 网络平台外来宠物贸易的现状}

与国内之前进行的线下市场贸易调查结果(梦 梦等, 2017)相比, 本次调查缺少部分雀形目和鹦形 目物种，如亚历山大鹦武(Psittacula eupatria)、太阳 雉尾鹦鹉(Aratinga solstitialis)、斑胸草雀(Taeniopygia guttata)等, 但增加了有鳞目物种。这显示出线 下市场和网络平台宠物贸易的差异, 网络平台大多 通过快递运输宠物, 这使得卖家出售的物种趋向于 体型小、活动频率和强度低、不易发出声响的龟鳖 目和有鳞目物种, 这两大类群是网络平台上外来宠 物贸易的主体。在线下市场贸易调查结果(梦梦等, 2017)的基础上，本次调查增加了11种被IUCN列为 极危和濒危 $(\mathrm{CR} / \mathrm{EN})$ 的物种和7种CITES附录I物种。 
这表明网络平台已经成为濒危保护动物贸易的重 要平台, 应当引起管理部门和社会各界的关注。

结合考虑物种的售卖信息条数和价格, 售卖信 息大于 5 条的 25 个物种中, 有 14 种的价格低于 1,000 元, 这显示网络平台上外来宠物的贸易主体是价格 低于 1,000 元的物种。有价格信息并被CITES列入附 录I和II的物种总共有 31 种，其中 $52 \%$ 的价格低于 1,000 元。综合来看, 价格低于 1,000 元的物种不仅售 卖规模较大同时也涉及较多保护物种, 管理部门应 加大对这部分物种的调查和监管力度。

本次调查共监测到 45 种 CITES 附录物种。 CITES是为了保护某些野生动植物物种不致由于国 际贸易而遭到过度开发利用而签订的, 但它的核心 在于管制而非完全禁止野生物种的国际贸易。为维 持濒危物种的野外种群并满足人类对物种的需求, 商业繁殖场对部分物种进行了人工繁育。对于可以 商业利用的人工繁殖第 2 代以上的CITES 附录物种, 根据《中华人民共和国野生动物保护法》的规定, 商 家应利用进口许可证、人工繁育许可证等相关证明, 从野生动物保护主管部门取得专用标识, 凭专用标 识出售和利用。由于网络信息获取的局限性, 很难 确定售卖物种个体的来源, 但有些卖家未提供物种 合法来源信息, 可能涉嫌非法买卖。本次调查到的 CITES附录物种中, 24种有圈养繁殖成功的案例(附 录1), 包括已经可以规模化商业繁殖的美洲镾蜥、 贝氏栉尾蚚(Ctenosaura bakeri)、真鳄龟(Macrochelys temminckii)等(Stephen et al, 2011; 杨君达, 2016), 这些物种直接来自野外的可能性较低。但繁 育技术不成功的物种, 来自野外的可能性则较高。

\section{2 网络平台外来宠物贸易造成的潜在危害}

宠物贸易是导致外来物种入侵的重要途径之 一(Hou et al, 2006)。目前已有一些外来物种在我国 形成了入侵种群, 对我国原生物种的生存发展造成 了威胁, 例如巴西红耳龟(Trachemys scripta elegans) 和牛蛙(Lithobates catesbeianus)。红耳龟的繁殖能力 极强, 与我国多种淡水龟栖息地重叠, 严重威胁到 我国本土龟类物种的生存(龚世平等, 2018)。牛蛙已 入侵我国广大区域, 可通过竞争、捕食和疾病传播 等多种方式危害我国本土两栖类。巴西红耳龟和牛 蛙已经被IUCN列为世界上最危险的 100 种外来入 侵物种(Cadi \& Joly, 2004; Hou et al, 2006)。

除了上述人们熟知的入侵种, 更多的潜在入侵
种也正在进入我国市场。如黑栉尾蚚和美洲髪蚚都 有成为入侵种的记录, 它们通过破坏建筑(Sementelli et al, 2008)、捕食当地物种(Krysko et al, 2003; Townsend et al, 2005)、竞争和杂交(Moss et al, 2018) 等方式对其入侵区域造成了社会、经济和生态威 胁。在加斯帕里利亚岛上, 黑栉尾蚚会与入侵物种 巴西胡椒树(Schinus terebinthifolius)形成互利关系, 达到大量繁殖的目的(Jackson \& Jackson, 2007)。科 研人员通过生态建模的方法预测评估出球蟒在美 国成为潜在外来入侵物种的风险极高(Reed, 2005)。 因为外来宠物的来源通常未知, 其潜在危害不容忽 视。外来宠物不仅会影响当地社会经济和生态的平 稳发展, 还会对人类的健康产生威胁(Derraik \& Phillips, 2010; Keller et al, 2011)。沙门氏菌感染的主 要来源是食物, 但在所有人类沙门氏菌病病例中, 估计有 $3 \%-5 \%$ 与接触外来宠物有关(Woodward et al, 1997)。许多两栖动物的皮肤会分泌有毒物质，对人 类有潜在的危害(Hou et al, 2006)。目前, 还没有关 于黑栉尾蜥、美洲鬛蜥和球蟒在我国的状况和作为 外来入侵种的风险评估。

宠物的大量需求会威胁到全球物种多样性, 走 私和非法贸易严重影响了濒危物种在原产地的种 群数量。Baker等(2013)发现2006-2011年之间发表 的关于野生动物贸易的报告和文献, 其中 $20 \%$ 是将 野生动物作为宠物或用于娱乐需求。在我国广州、 北京、上海等城市开展的市场贸易调查都发现有列 入CITES附录的动物被售卖, 但其中有些是已经规 模化人工商业养殖的、根据CITES公约可以合法贸 易的物种(如暹罗鳄、真鳄龟等)(Cheung \& Dudgeon, 2006; 梦梦等, 2017; 王健等, 2018)。龟类作为宠物、 肉类和传统药物的市场规模非常大, 而且还在不断 扩大, 这对全球野生龟类的生存产生了极大的威胁 (Mendiratta et al, 2017)。中国亚洲龟类贸易调查发 现, 在中国南方有贸易的157种龟类中, 有155种是 作为宠物出售的(Cheung \& Dudgeon, 2006)。宠物贸 易还导致了大量鹦绒跨境贩运, 使其成为CITES附 录中贸易最频繁的鸟类之一(Fogell et al, 2018)。例 如非洲灰鹦武弗期以来在野外被大量捕获, 用于国 际间的宠物鸟类贸易, 其在中西非的种群数量不断 减少(Bush et al, 2014), 野生种群遭受了严重的威胁 (Martin et al, 2018)。 


\section{3 保护管理建议}

\subsection{1 落实法律法规, 建立完善网络平台动物贸易 监测和管理机制}

建议对网络平台动物贸易进行持续性的全面 调查，同时应建立预防动物非法贸易的管控机制, 积极在公安、海关、科研机构、民间组织等机构之 间进行多方合作, 提升工作人员的动物保护意识和 专业度。建议在网络平台上利用图片识别、大数据、 人工智能等技术, 实时监控并捕捉涉嫌非法动物贸 易的信息, 将信息及时上报相关部门, 同时注销涉 嫌非法贸易的用户。还应在网络平台上建立全员监 管机制, 拓宽群众举报渠道, 引入社会监督。建议 主管部门严格执行《中华人民共和国野生动物保护 法》和《濒危野生动植物进出口管理条例》, 加强 国家重点保护物种和CITES附录物种管理。细化动 物贸易的相关法律解释, 从网络、实体市场、运输 (快递)等多个环节进行监管, 细化管理措施, 落实 责任, 以杜绝网络平台的动物非法贸易行为。

\subsection{2 构建外来物种监管制度体系, 系统调研外来} 物种对公众健康和中国生态系统的影响

宠物贸易是导致外来物种入侵的重要途径之 一(Hulme et al, 2008)。这些外来物种的贸易不仅威 胁着该物种在原产国的生存, 也对我国的生态系统 有着潜在的威胁。因此, 建议相关部门与科研机构 联合, 系统调研评估外来物种对中国生态系统的影 响, 公布可能具有入侵风险的动物外来物种名录。 主管部门应结合研究结果, 从我国外来物种入侵事 件的发生特点、目前状况、未来趋势等多方面考虑, 尽快建立有效的外来物种监管制度体系。并根据相 关法律法规, 从来源、市场、运输、野外多方面对 外来物种进行严格监管, 对于违法违规行为, 按照 法律规定严厉查处。

\subsection{3 加大宣传教育, 提升公众的认知和保护水平}

网络平台上存在大量个人繁育外来物种进行 买卖、弃养转卖的现象, 说明公众对濒危保护物种 和外来物种的相关知识缺乏了解。建议有关部门加 强关于濒危保护动物和外来物种的宣传教育和科 学知识普及工作, 明确合法贸易物种, 指导公众了 解濒危野生动物和外来物种贸易可能带来的健康 问题和生态风险, 提高公众的动物保护意识, 减少 饲养野生动物和外来物种作为宠物的行为。此外, 建议教育部门和学校加强对学生的动物保护、物种
入侵等相关法律知识教育工作。

\section{参考文献}

Annorbah NND, Collar NJ, Marsden SJ (2016) Trade and habitat change virtually eliminate the grey parrot Psittacus erithacus from Ghana. IBIS, 158, 82-91.

Baker SE, Cain R, Kesteren F, Zommers ZA, D’Cruze N, Macdonald DW (2013) Rough trade: Animal welfare in the global wildlife trade. BioScience, 63, 928-938.

Bush ER, Baker SE, Macdonald DW (2014) Global trade in exotic pets 2006-2012: Exotic pet trade. Conservation Biology, 28, 663-676.

Cadi A, Joly P (2004) Impact of the introduction of the red-eared slider (Trachemys scripta elegans) on survival rates of the European pond turtle (Emys orbicularis). Biodiversity and Conservation, 13, 2511-2518.

Carrete M, Tella JL (2008) Wild-bird trade and exotic invasions: A new link of conservation concern? Frontiers in Ecology and the Environment, 6, 207-211.

Cheung SM, Dudgeon D (2006) Quantifying the Asian turtle crisis: Market surveys in southern China, 2000-2003. Aquatic Conservation: Marine and Freshwater Ecosystems, 16, 751-770.

CITES (The Convention on International Trade in Endangered Species of Wild Fauna and Flora) (2019) Checklist of CITES species. http://checklist.cites.org/. (accessed on 2020-04-28)

Derraik JGB, Phillips S (2010) Online trade poses a threat to biosecurity in New Zealand. Biological Invasions, 12, 1477-1480.

Fogell DJ, Martin RO, Bunbury N, Lawson B, Sells J, McKeand AM, Tatayah V, Trung CT, Groombridge JJ (2018) Trade and conservation implications of new beak and feather disease virus detection in native and introduced parrots. Conservation Biology, 32, 1325-1335.

Gong SP, Chow AT, Fong JJ, Shi HT (2009) The chelonian trade in the largest pet market in China: Scale, scope and impact on turtle conservation. Oryx, 43, 213-216.

Gong SP, Wang JC, Shi HT, Song RH, Xu RMI (2006) Illegal trade and conservation requirements of freshwater turtles in Nanmao, Hainan Province, China. Oryx, 40, 331-336.

Gong SP, Yang JB, Ge Y, Gaillard D (2018) Extent and mechanisms of the increasing geographic distribution of the alien red-eared slider (Trachemys scripta elegans) in China. Chinese Journal of Wildlife, 39, 373-378. (in Chinese with English abstract) [龚世平, 杨江波, 葛研, Gaillard D (2018) 外来物种红耳龟在中国野外分布现状及扩散路径 研究. 野生动物学报, 39, 373-378.]

Harkins GW, Martin DP, Christoffels A, Varsani A (2014) Towards inferring the global movement of beak and feather disease virus. Virology, 450, 24-33.

Harrison JR, Roberts DL, Hernandez-Castro J (2016) Assessing the extent and nature of wildlife trade on the dark web. Conservation Biology, 30, 900-904. 
Hou PCL, Shiau TW, Tu MC, Chen CC, Chen TY, Tsai YF, Lin CF, Wu SH (2006) Exotic amphibians in the pet shops of Taiwan. Taiwania, 51, 87-92.

Hulme PE, Bacher S, Kenis M, Klotz S, Kühn I, Minchin D, Nentwig W, Olenin S, Panov V, Pergl J, Pyšek P, Roques A, Sol D, Solarz W, Vilà M (2008) Grasping at the routes of biological invasions: A framework for integrating pathways into policy. Journal of Applied Ecology, 45, 403-414.

IUCN (International Union for Conservation of Nature) (2017) IUCN Red List of Threatened Species. http://www.iucnredlist. org/. (accessed on 2019-08-30)

Jackson JA, Jackson BJS (2007) An apparent mutualistic association between invasive exotics: Brazilian pepper Schinus terebinthifolius and black spiny-tailed iguanas Ctenosaura similis. Natural Areas Journal, 27, 254-257.

Jensen TJ, Auliya M, Burgess ND, Aust PW, Pertoldi C, Strand $\mathrm{J}$ (2019) Exploring the international trade in African snakes not listed on CITES: Highlighting the role of the internet and social media. Biodiversity and Conservation, 28, 1-19.

Keller RP, Geist J, Jeschke JM, Kühn L (2011) Invasive species in Europe: Ecology, status, and policy. Environmental Sciences Europe, 23, 23-40.

Krysko KL, King FW, Enge KM, Reppas AT (2003) Distribution of the introduced black spiny-tailed iguana Ctenosaura similis on the southwestern coast of Florida. Florida Scientist, 66, 74-79.

Lockwood JL (2017) Exotic birds provide unique insight into species invasions. Proceedings of the National Academy of Sciences, USA, 114, 9237-9239.

Ma B, Jin HY, Pei EL (2013) Status and countermeasures for live wildlife trade on the Internet in Shanghai City. Chinese Journal of Wildlife, 34, 46-49. (in Chinese with English abstract) [马波, 金慧宇, 裴恩乐 (2013) 上海市利用互联 网交易珍稀活体野生动物现状及对策研究. 野生动物, 34, 46-49.]

Martin RO, Senni C, D’Cruze NC (2018) Trade in wild-sourced African grey parrots: Insights via social media. Global Ecology and Conservation, 15, e00429.

Mendiratta U, Sheel V, Singh S (2017) Enforcement seizures reveal large-scale illegal trade in India's tortoises and freshwater turtles. Biological Conservation, 207, 100-105.

Meng M, Ma JZ, Ji JW, Chen WH, Wang ZK, Yin F (2018) Dynamic analysis of the import and export trade of endangered wild animals in China. Forest Resources Management, (3), 19-24. (in Chinese with English abstract) [梦梦, 马建 章, 纪建伟, 陈文汇, 王忠昆, 尹峰 (2018) 我国野生动 物进出口贸易动态与对策分析. 林业资源管理, (3), 19-24.]

Meng M, Ma JZ, Yin F, Chen WH, Ji JW (2017) Investigation of the current trade situation of alien vertebrate species in China and analysis of corresponding management strategies. Biodiversity Science, 25, 1137-1143. (in Chinese with English abstract) [梦梦, 马建章, 尹峰, 陈文汇, 纪建伟
(2017) 我国典型城市外来野生脊椎动物贸易状况及管理 对策. 生物多样性, 25, 1137-1143.]

Moorhouse TP, Balaskas M, D’Cruze NC, Macdonald DW (2017) Posts could reduce consumer demand for exotic pets. Conservation Letters, 10, 337-345.

Moss JB, Welch ME, Burton FJ, Vallee MV, Houlcroft EW, Laaser T, Gerber GP (2018) First evidence for crossbreeding between invasive Iguana iguana and the native rock iguana (Genus Cyclura) on Little Cayman Island. Biological Invasions, 20, 817-823.

Reed RN (2005) An ecological risk assessment of nonnative boas and pythons as potentially invasive species in the United States. Risk Analysis, 25, 753-766.

Roskov Y, Ower G, Orrell T, Nicolson D, Bailly N, Kirk PM, Bourgoin T, Dewalt RE, Decock W, Nieukerken E, Zarucchi J, Penev L (2019) Species 2000 \& ITIS Catalogue of Life, 2019 Annual Checklist. http://www.catalogueoflife.org/annualchecklist/2019/. (accessed on 2019-08-24)

Sementelli A, Smith HT, Meshaka WE, Engeman RM (2008) Just green iguanas? The associated costs and policy implications of exotic invasive wildlife in South Florida. Public Works Management and Policy, 12, 599-606.

Shepherd CR, Nijman V, Krishnasamy K, Eaton JA, Chng SCL (2016) Illegal trade pushing the critically endangered black-winged myna Acridotheres melanopterus towards imminent extinction. Bird Conservation International, 26, 147-153.

Shi HT (2008) Identification Manual for Traded Turtles in China. Encyclopedia of China Publishing House, Beijing. (in Chinese) [史海涛 (2008) 中国贸易龟类检索图鉴. 中 国大百科全书出版社, 北京.]

Siriwat P, Nekaris KA, Nikman V (2019) The role of the anthropogenic Allee effect in the exotic pet trade on Facebook in Thailand. Journal for Nature Conservation, 51, 125726.

Siriwat P, Nijman V (2018) Illegal pet trade on social media as an emerging impediment to the conservation of Asian otters species. Journal of Asia-Pacific Biodiversity, 11, 469-475.

Stephen C, Pasachnik S, Reuter A, Mosig P, Ruyle L, Fitzgerald L (2011) Survey of Status, Trade, and Exploitation of Central American Iguanas. Department of Interior, United States Fish and Wildlife Service, Washington, DC.

Su S, Cassey P, Blackburn TM (2016) The wildlife pet trade as a driver of introduction and establishment in alien birds in Taiwan. Biological Invasions, 18, 215-229.

Sung Y, Fong JJ (2018) Assessing consumer trends and illegal activity by monitoring the online wildlife trade. Biological Conservation, 227, 219-225.

Townsend JH, Slapcinsky J, Krysko KL, Donan EM, Golden EA (2005) Predation of a tree snail Drymaeus multilineatus (Gastropoda: Bulimulidae) by Iguana iguana (Reptilia: Iguanidae) on Key Biscayne. Southeastern Naturalist, 4, 361-364.

Wang J, Qi S, Guo CP, Lin CR, Xiao JJ, Wang YY, Lin SS 
(2018) Survey on amphibian pet trade in Huadiwan market in Guangzhou. Chinese Journal of Zoology, 53, 61-68. (in Chinese with English abstract) [王健, 齐硕, 郭淳鹏, 林灿 荣, 肖嘉杰, 王英永, 林石狮 (2018) 广州市花地湾市场 宠物两栖动物调查. 动物学杂志, 53, 61-68.]

Wang S, Xie Y, Wang JJ (2001) A Dictionary of Mammalian Names (Latin Chinese English). Hunan Education Press, Changsha. [汪松, 解炎, 王嘉骏 (2001) 世界哺乳动物名 典(拉汉英). 湖南教育出版社, 长沙.]

Woodward DL, Khakhria R, Johnson WM (1997) Human salmonellosis associated with exotic pets. Journal of Clinical Microbiology, 35, 2786-2790.

Xiong XQ, You WX, Zhang L, Li YL, Li K (2017) Status of illegal use of wildlife resources in Beijing. Chinese Journal of Wildlife, 38, 376-385. (in Chinese with English abstract) [熊小倩, 尤炜轩, 张玲, 李又霖, 李凯 (2017) 北京地区 野生动物资源非法利用状况分析. 野生动物学报, 38, 376-385.]

Yang JD (2016) Artificial breeding method of Chelydra serpentina osceola. China, CN103843723B, 2016-06-08. (in Chinese) [杨君达 (2016) 一种鳄龟人工繁殖方法. 中国, CN103843723B, 2016-06-08.]

Ye YC, Yu WH, Newman C, Buesching CD, Xu YL, Xiao X, Macdonald DW, Zhou ZM (2020) Effects of regional economics on the online sale of protected parrots and turtles in China. Conservation Science and Practice, 2, e161.

Zhao EM, Jiang YM, Huang QY, Hu SQ, Fei L, Ye CY (1993) Latin-Chinese-English Names of Amphibians and Reptiles. Science Press, Beijing. [赵尔宓, 江跃明, 黄庆云, 胡淑琴, 费梁, 叶昌媛 (1993) 拉汉英两栖爬行动物名称. 科学出 版社, 北京.]

Zheng GM (2002) A Checklist on the Classification and Distribution of the Birds of the World. Science Press, Beijing. (in Chinese) [郑光美 (2002) 世界鸟类分类与分 布名录. 科学出版社, 北京.]

(责任编委: 蒋志刚 责任编辑: 问文杰)

\section{附录 Supplementary Material}

附录1 网络平台贸易外来宠物名录

Appendix 1 List of species of exotic pets traded on the internet http://www.biodiversity-science.net/fileup/PDF/2019391-1.pdf 
附录1 网络平台贸易外来宠物名录

Appendix 1 List of species of exotic pets traded on the internet

\begin{tabular}{|c|c|c|c|c|c|c|c|}
\hline \multirow{4}{*}{ 纲/目 } & \multirow[b]{3}{*}{ 科 } & \multirow[b]{3}{*}{ 物种 } & \multirow{4}{*}{ 市场名 } & CITES 级别, (圈养繁殖成功 & & \multirow{4}{*}{ 价格 Price } \\
\hline & & & & 案例) & IUCN 红 & & \\
\hline & & & & \multirow{5}{*}{$\begin{array}{l}\text { Protection level } \\
\text { of CITES, (Successful cases }\end{array}$} & 色名录 & 数量 & \\
\hline & & & & & & & \\
\hline \multirow[t]{4}{*}{ Class/Order } & Family & Species & Trade name & & IUCN & Number & (RMB Yuan) \\
\hline & & & & & & & \\
\hline & & & & & \multirow{2}{*}{\multicolumn{2}{|c|}{ Red List }} & \\
\hline & & & & of captive breeding) & & & \\
\hline \multicolumn{8}{|l|}{ 哺乳纲 Mammalia } \\
\hline 啮齿目 Rodentia & 松鼠科 Sciuridae & 黑尾草原犬鼠 Cynomys ludovicianus & 黑尾土拨鼠 & & $\mathrm{LC}$ & 4 & ++ \\
\hline 袋鼠目 Diprotodontia & 袋鼣科 Petauridae & 蜜袋䶂 Petaurus breviceps & 蜜袋䶂 & & $\mathrm{LC}$ & 1 & + \\
\hline \multicolumn{8}{|l|}{ 爬行纲 Reptilia } \\
\hline 鳄目 Crocodylia & 䲞科 Crocodylidae & 暹罗魾 Crocodylus siamensis & 暹罗鲇 & I, (Platt et al, 2012) & $\mathrm{CR}$ & 3 & + \\
\hline \multirow[t]{7}{*}{ 龟鳖目 Testudines } & 侧颈龟科 Chelidae & 枯叶侧颈龟 Chelus fimbriata & 枯叶龟 & & & 2 & ++ \\
\hline & & 红腹侧颈龟 Emydura subglobosa & 圆澳龟 & & $\mathrm{LC}$ & 2 & \\
\hline & 地龟科 Geoemydidae & 马来龟 Malayemys subtrijuga & 马来食螺龟 & II & VU & 2 & + \\
\hline & & 安南摄龟 Mauremys annamensis & 安南龟 & I, (Uhrig \& Lee, 2006) & $\mathrm{CR}$ & 2 & + \\
\hline & & 印度泛棱背龟 Pangshura tecta & 印度棱背龟 & I, (Vyas, 2001) & $\mathrm{LC}$ & 4 & + \\
\hline & 动胸龟科 Kinosternidae & 窄桥匣子龟 Claudius angustatus & 窄桥膺香龟 & & NT & 1 & ++ \\
\hline & & 斑纹动胸龟 Kinosternon acutum & 斑纹泥龟 & & NT & 1 & \\
\hline
\end{tabular}




\begin{tabular}{|c|c|c|c|c|c|c|}
\hline & 大瘪香龟 Staurotypus triporcatus & 墨西哥榭香龟 & & NT & 5 & + \\
\hline & 剃刀动胸龟 Sternotherus carinatus & 刀背廭动胸龟 & & $\mathrm{LC}$ & 3 & + \\
\hline \multirow[t]{11}{*}{ 陆龟科 Testudinidae } & 辐纹陆龟 Astrochelys radiata & 辐射陆龟 & I, (Hammer, 2015) & $\mathrm{CR}$ & 6 & ++ \\
\hline & 喉盾陆龟 Astrochelys yniphora & 安哥洛卡象龟 & I, (López et al, 2017) & $\mathrm{CR}$ & 2 & ++++ \\
\hline & 塞舌尔巨陆龟 Aldabrachelys gigantea & 亚达伯拉象龟 & II & $\mathrm{VU}$ & 4 & \\
\hline & 胫刺陆龟 Centrochelys sulcata & 苏卡达象龟 & II, (Stearns, 2007) & $\mathrm{VU}$ & 19 & ++ \\
\hline & 黄腿陆龟 Chelonoidis denticulata & 黄腿陆龟 & II & $\mathrm{VU}$ & 5 & + \\
\hline & 印度星龟 Geochelone elegans & 印度星斑陆龟 & I, (Vyas, 2005) & VU & 8 & + \\
\hline & 缅甸星龟 Geochelone platynota & 缅甸星龟 & I, (Gerald et al, 2012) & $\mathrm{CR}$ & 7 & ++ \\
\hline & 黑凹甲陆龟 Manouria emys & 黑靴陆龟 & II & $\mathrm{CR}$ & 1 & \\
\hline & 豹纹陆龟 Stigmochelys pardalis & 豹纹陆龟 & II & $\mathrm{LC}$ & 16 & + \\
\hline & 希腊陆龟 Testudo graeca & 欧洲陆龟 & II, (Robinzon et al, 2005) & VU & 2 & + \\
\hline & 赫氏陆龟 Testudo hermanni & 赫曼龟 & II, (Kirsche, 1984) & NT & 5 & + \\
\hline \multirow[t]{5}{*}{ 泽龟科 Emydidae } & 点斑水龟 Clemmys guttata & 星点水龟 & II, (Cassim, 2006) & EN & 1 & ++ \\
\hline & 网目鸡龟 Deirochelys reticularia & 东部网目鸡龟 & & & 1 & \\
\hline & 木雕水龟 Glyptemys insculpta & 木雕水龟 & II & EN & 1 & \\
\hline & 得克萨斯图龟 Graptemys versa & 德州地图龟 & III & $\mathrm{LC}$ & 1 & ++ \\
\hline & 菱斑龟 Malaclemys terrapin & 图钻龟 & II & VU & 3 & ++ \\
\hline
\end{tabular}




\begin{tabular}{|c|c|c|c|c|c|c|c|}
\hline & & 卡罗林那箱龟 Terrapene carolina & 卡罗莱纳箱龟 & II & $\mathrm{VU}$ & 2 & ++ \\
\hline & & 红耳龟 Trachemys scripta elegans & 巴西红耳龟 & & $\mathrm{LC}$ & 11 & + \\
\hline & 鳄龟科 Chelydridae & 大魾龟 Macrochelys temminckii & 真鲇龟 & III, (DiGeronimo et al, 2018) & $\mathrm{VU}$ & 6 & ++ \\
\hline & & 蛇鳄龟 Chelydra serpentina & 拟魾龟 & III, (Yang et al, 2016) & $\mathrm{LC}$ & 7 & + \\
\hline \multirow[t]{14}{*}{ 有鳞目 Squamata } & 安乐蜥科 Dactyloidae & 绿安乐蜥 Anolis carolinensis & 安乐蜥 & & $\mathrm{LC}$ & 7 & + \\
\hline & 澳虎科 Diplodactylidae & 纤毛多趾虎 Correlophus ciliatus & 睫角守宫 & & $\mathrm{VU}$ & 14 & + \\
\hline & & 北方瘤尾虎 Oedura castelnaui & 北部丝绒守宫 & & $\mathrm{LC}$ & 1 & + \\
\hline & & 耳多趾虎 Rhacodactylus auriculatus & 盖勾亚守宫 & & $\mathrm{LC}$ & 2 & +++ \\
\hline & & 多趾虎 Rhacodactylus leachianus & 巨人守宫 & & $\mathrm{LC}$ & 5 & ++ \\
\hline & 壁虎科 Gekkonidae & 尾疹残趾虎 Phelsuma laticauda & 马达加斯加金粉 & II & $\mathrm{LC}$ & 4 & + \\
\hline & & & 守宫 & & & & \\
\hline & & 三带褶虎 Ptychozoon trinotaterra & 飞蹼守宫 & & $\mathrm{LC}$ & 1 & + \\
\hline & 避役科 Chamaeleonidae & 盔甲避役 Chamaeleo calyptratus & 高冠变色龙 & II, (Diaz et al, 2015) & $\mathrm{LC}$ & 6 & + \\
\hline & & 颈盾避役 Chamaeleo dilepis & 喷点变色龙 & II & $\mathrm{LC}$ & 1 & \\
\hline & & 豹纹避役 Furcifer pardalis & 七彩变色龙 & II, (Ferguson et al, 2002) & $\mathrm{LC}$ & 6 & \\
\hline & & 尖嘴避役 Trioceros jacksonii & 杰克森变色龙 & II & $\mathrm{LC}$ & 3 & + \\
\hline & 鞭尾蜥科 Teiidae & 臼齿蜥 Ameiva ameiva & 绿从林蜊 & & $\mathrm{LC}$ & 1 & + \\
\hline & & 条纹鞭尾蜊 Cnemidophorus lemniscatus & 彩虹鞭尾蜥 & & & 1 & + \\
\hline
\end{tabular}




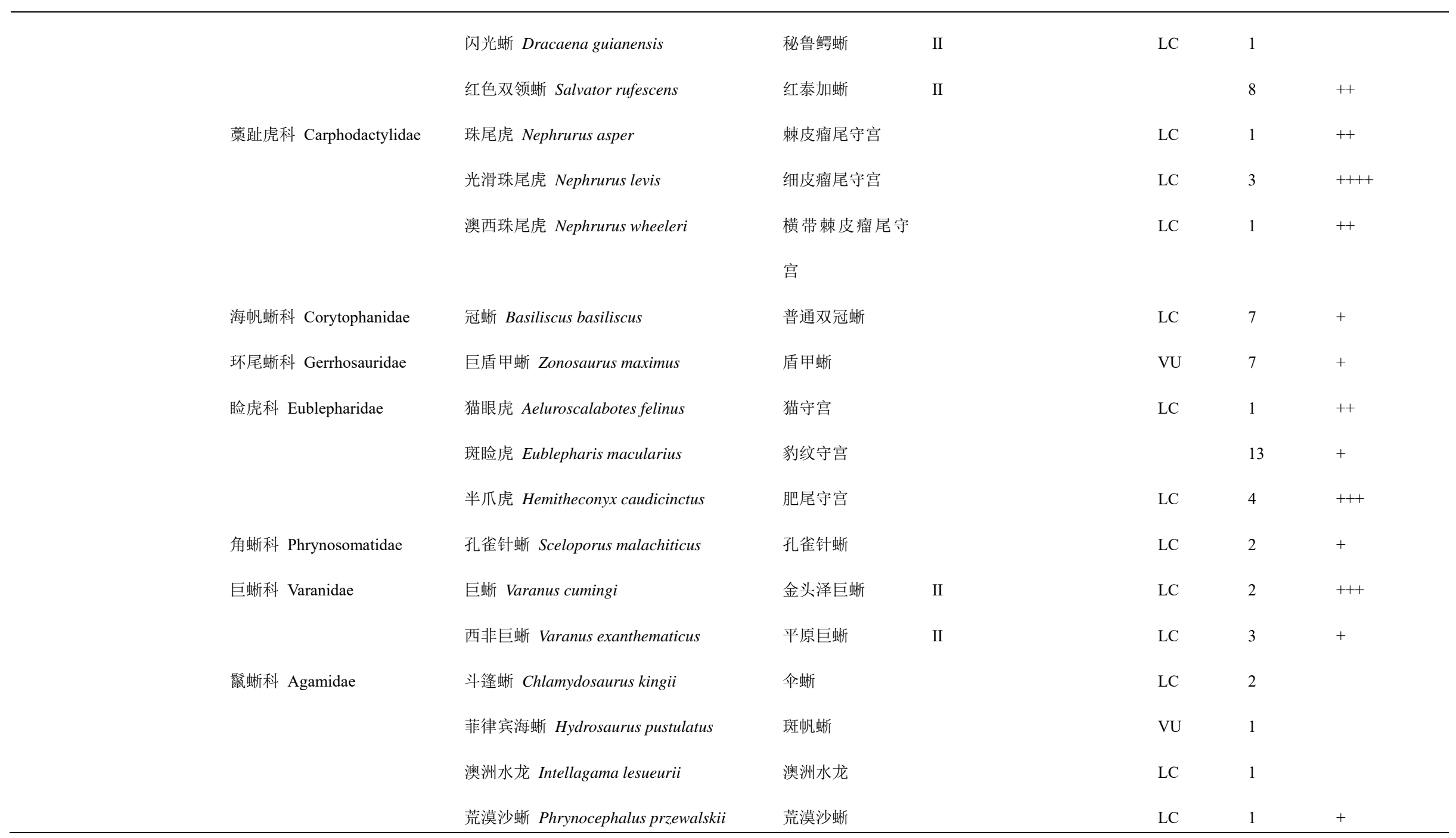




\begin{tabular}{|c|c|c|c|c|c|c|}
\hline & 䯿蜥 Pogona vitticeps & 鬃狮蜥 & & LC & 26 & + \\
\hline & 眼斑刺尾蜊 Uromastyx ocellata & 孔雀王者蜥 & II & LC & 2 & \\
\hline \multirow[t]{7}{*}{ 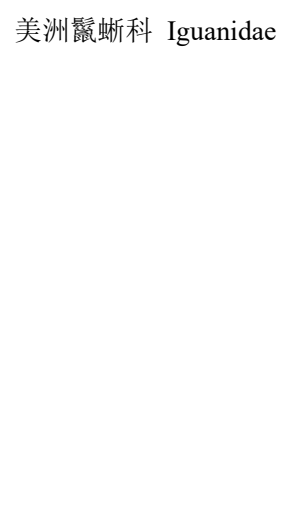 } & 贝氏栉尾蜥 Ctenosaura bakeri & 乌蒂拉栉尾蜥 & II, (Stephen et al, 2011) & $\mathrm{CR}$ & 3 & \\
\hline & 危地马拉栉尾蜥 Ctenosaura palearis & 危地马拉刺尾镾 & II & $\mathrm{EN}$ & 1 & \\
\hline & & 蜥 & & & & \\
\hline & 五棱栉尾蚚 Ctenosaura quinquecarinata & 狼牙棒刺尾瓺蜥 & II, (Stephen et al, 2011) & $\mathrm{EN}$ & 1 & + \\
\hline & 黑栉尾蚚 Ctenosaura similis & 中美洲刺尾瓺蜥 & II, (Stephen et al, 2011) & $\mathrm{LC}$ & 33 & ++ \\
\hline & 蓝岩鬛蜥 Cyclura lewisi & 蓝岩鬛蜥 & I, (Grant \& Hudson, 2014) & $\mathrm{EN}$ & 3 & ++ \\
\hline & 美洲鬛蜘 Iguana iguana & 绿鬛蜥 & II, (Stephen et al, 2011) & LC & 32 & + \\
\hline \multirow[t]{5}{*}{ 石龙子科 Scincidae } & 菲南波多火蜥 Lepidothyris fernandi & 火焰石龙子 & & & 3 & \\
\hline & 沙鱼蜊 Scincus scincus & 砂鱼蜥 & & & 1 & + \\
\hline & 巨柔蜓 Tiliqua gigas & 巨型蓝舌石龙子 & & & 1 & ++ \\
\hline & 蓝色柔蜊 Tiliqua scincoides & 印尼蓝舌石龙子 & & LC & 6 & ++ \\
\hline & 细三棱蜥 Tribolonotus gracilis & 红眼鹰蜥 & & LC & 2 & + \\
\hline 蜊蜴科 Lacertidae & 蓝斑蜘蜴 Timon lepidus & 珠宝蜥 & & NT & 2 & ++ \\
\hline 项圈蜘科 Crotaphytidae & 项圈蜥 Crotaphytus collaris & 项圈蜥 & & $\mathrm{LC}$ & 2 & + \\
\hline 眼镜蛇科 Elapidae & 泰国眼镜蛇 Naja siamensis & 中南半岛射毒眼 & II & $\mathrm{VU}$ & 2 & \\
\hline & & 镜蛇 & & & & \\
\hline
\end{tabular}




\begin{tabular}{|c|c|c|c|c|c|c|}
\hline 鳗形蛇科 Lamprophiidae & 家蛇 Boaedon fuliginosus & \multicolumn{3}{|l|}{ 非洲褐屋蛇 } & 1 & + \\
\hline 美洲钝头蛇科 Dipsadidae & 西猪鼻蛇 Heterodon nasicus & \multicolumn{2}{|l|}{ 猪鼻蛇 } & $\mathrm{LC}$ & 1 & + \\
\hline 叶趾虎科 Phyllodactylidae & 墙虎 Tarentola chazaliae & \multicolumn{2}{|l|}{ 头盔守宫 } & $\mathrm{VU}$ & 1 & ++ \\
\hline & 守宫 Tarentola mauritanica & \multicolumn{2}{|l|}{ 鳄鱼守宫 } & $\mathrm{LC}$ & 1 & + \\
\hline 游蛇科 Colubridae & 加州王蛇 Lampropeltis getula californiae & \multicolumn{2}{|l|}{ 加州王蛇 } & $\mathrm{LC}$ & 2 & ++ \\
\hline & 墨西哥王蛇 Lampropeltis mexicana & \multicolumn{2}{|l|}{ 墨西哥王蛇 } & $\mathrm{LC}$ & 3 & ++ \\
\hline & 高山王蛇 Lampropeltis pyromelana & \multicolumn{2}{|l|}{ 高山王蛇 } & $\mathrm{LC}$ & 1 & ++ \\
\hline & 乳王蛇 Lampropeltis triangulum & \multicolumn{2}{|l|}{ 奶蛇 } & $\mathrm{LC}$ & 7 & \\
\hline & 洪都拉斯乳王蛇 L. t.hondurensis & \multicolumn{2}{|l|}{ 洪都拉斯奶蛇 } & & & \\
\hline & 纳尔逊乳王蛇 L.t. nelsoni & \multicolumn{2}{|l|}{ 纳尔逊奶蛇 } & & & \\
\hline & 帕布拉乳王蛇 L. t. campbelli & \multicolumn{2}{|l|}{ 帕布拉奶蛇 } & & & \\
\hline & 玉米锦蛇 Pantherophis guttatus & \multicolumn{2}{|l|}{ 玉米蛇 } & $\mathrm{LC}$ & 18 & ++ \\
\hline & 黑唇牛蛇 Pituophis melanoleucus & \multicolumn{2}{|l|}{ 北美松蛇 } & $\mathrm{LC}$ & 3 & ++ \\
\hline \multirow[t]{2}{*}{ 蟒科 Pythonidae } & 绿树蟒 Morelia viridis & 绿树蟒 & II, (Lyons, 2011) & $\mathrm{LC}$ & 4 & ++ \\
\hline & 球蟒 Python regius & 球蟒 & II, (Martin, 1998) & $\mathrm{LC}$ & 13 & + \\
\hline \multirow[t]{2}{*}{ 蚰科 Boidae } & 巨蚰 Boa constrictor & 红尾蚰 & II & EN & 3 & ++ \\
\hline & 班尼水蚛 Eunectes beniensis & 亚马逊森蚰 & II & $\mathrm{LC}$ & 1 & \\
\hline 蝰科 Viperidae & 犀角哏蝰 Bitis rhinoceros & 犀角啷蝰 & & $\mathrm{LC}$ & 1 & \\
\hline
\end{tabular}




\begin{tabular}{|c|c|c|c|c|c|c|}
\hline & & 阿拉伯角蛏 Cerastes gasperettii & 沙漠角蛏 & $\mathrm{LC}$ & 1 & \\
\hline & & 紫棕烙铁头 & 红树林竹叶青 & $\mathrm{LC}$ & 1 & \\
\hline & & Trimeresurus purpureomaculatus & & & & \\
\hline & & 黑绿烙铁头 Tropidolaemus wagleri & 韦氏竹叶青 & $\mathrm{LC}$ & 2 & \\
\hline \multirow[t]{12}{*}{ 无尾目 Anura } & 蟾蜍科 Bufonidae & 海蟾蜍 Rhinella marina & 巨型海蟾蜍 & $\mathrm{LC}$ & 2 & + \\
\hline & 短头蛙科 Brevicipitidae & 散疮短头蛙 Breviceps adspersus & 散疮短头蛙 & $\mathrm{LC}$ & 1 & + \\
\hline & 姬蛙科 Microhylidae & 双条螳螂 Phrynomantis bifasciatus & 红带步行蛙 & $\mathrm{LC}$ & 1 & + \\
\hline & 角蟾科 Megophryidae & 尖吻角蟾 Megophrys nasuta & 枯叶蛙 & $\mathrm{LC}$ & 1 & \\
\hline & 角花蟾科 Ceratophryidae & 显角花蟾 Ceratophrys cornuta & 亚马逊角蛙 & $\mathrm{LC}$ & 4 & + \\
\hline & & 克氏角花蟾 Ceratophrys cranwelli & 钟角蛙 & $\mathrm{LC}$ & 6 & + \\
\hline & & 圆眼珍珠蛙 Lepidobatrachus laevis & 猫眼珍珠蛙 & LC & 3 & + \\
\hline & 箱头蛙科 Pyxicephalidae & 非洲牛箱头蛙 Pyxicephalus adspersus & 非洲牛箱头蛙 & $\mathrm{LC}$ & 5 & + \\
\hline & 叶泡蛙科 Phyllomedusidae & 索瓦叶泡蛙 Phyllomedusa sauvagii & 蜡白猴树蛙 & $\mathrm{LC}$ & 1 & \\
\hline & 雨蛙科 Hylidae & 绿雨滨蛙 Litoria caerulea & 老爷树蛙 & $\mathrm{LC}$ & 5 & + \\
\hline & & 亚马逊䊁头蛙 & 亚马逊牛奶蛙 & $\mathrm{LC}$ & 1 & + \\
\hline & & Trachycephalus resinifictrix & & & & \\
\hline 有尾目 Caudata & 钝口螈科 Ambystomatidae & 虎纹针口螈 Ambystoma tigrinum & 虎纹蝾螈 & $\mathrm{LC}$ & 2 & + \\
\hline 回纲 Aves & & & & & & \\
\hline
\end{tabular}




\begin{tabular}{|c|c|c|c|c|c|c|c|c|}
\hline \multirow[t]{3}{*}{ 鹦形目 } & Psittaciformes & 凤头鹦鹉科 Cacatuidae & 鸡尾鹦武 Nymphicus hollandicus & 玄凤鹦鹉 & & LC & 3 & + \\
\hline & & 鸭武科 Psittacidae & 绯红金刚鹦鹉 Ara macao & 五彩金刚鹦武 & I, (Figueras, 2013) & $\mathrm{LC}$ & 3 & +++ \\
\hline & & & 非洲灰鹦鹉 Psittacus erithacus & 非洲灰鹦鹉 & I, (Martin, 2018) & EN & 8 & ++ \\
\hline
\end{tabular}

+1-999元; ++ 1,000-4,999元; +++5,000-9,999元; ++++>10,000元。

Cassim K (2006) The Effectiveness of Captive Release Conservation Methods for Spotted Turtles (Clemmys guttata). Rochester Institute of Technology, New York.

Diaz RE, Anderson CV, Baumann DP, Kupronis R, Jewell D, Piraquive C, Kupronis J, Winter K, Greek TJ, Trainor PA (2015) Captive care, raising, and breeding of the veiled chameleon (Chamaeleo calyptratus). CSH Protocols, 2015, 943-949.

DiGeronimo PM, Girolamo ND, Grasperge BJ, Gregory BB, Jowett P, Nevarez JG (2018) Assessment of blood lead, zinc, and mercury concentrations and cholinesterase activity in captive-reared alligator snapping turtles (Macrochelys temminckii) in Louisiana, USA. Journal of Wildlife Diseases, $54,553-557$.

Ferguson GW, Gehrmann WH, Chen TC, Dierenfeld ES, Holick MF (2002) Effects of artificial ultraviolet light exposure on reproductive success of the female panther chameleon (Furcifer pardalis) in captivity. Zoo Biology, 21, 525-537.

Figueras RR (2013) Scarlet macaw Ara macao cyanoptera conservation programme in Mexico. International Zoo Yearbook, 48, 48-60.

Gerald K, Eric G, Peter P (2012) Endoscopic imaging of gonads, sex ratio and temperature dependent sex determination in captive bred juvenile Burmese star tortoises Geochelone platynota. Asian Herpetological Research, 2, 240-244.

Grant TD, Hudson RD (2014) West Indian iguana Cyclura spp reintroduction and recovery programmes: Zoo support and involvement. International Zoo Yearbook, $49,49-55$

Hammer JM (2015) Reproduction as a function of living conditions: The breeding biology of the radiated tortoise (Astrochelys radiata) under natural and captive 
conditions in southwest Madagascar. Journal of Herpetology, 49, 633-640.

Kirsche W (1984) An F2-generation of Testudo hermanni hermanni GMELIN bred in captivity with remarks on the breeding of Mediterranean Tortoises $1976-1981$. Amphibia-Reptilia, 5, 31-35.

Lyons JA (2011) Wildlife laundering through breeding farms: Illegal harvest, population declines and a means of regulating the trade of green pythons (Morelia viridis) from Indonesia. Biological Conservation, 144, 3073-3081.

López J, Waters M, Routh A, Rakotonanahary TF, Woolaver L, Thomasson A, Holmes E, Steinmetz HW (2017) Hematology and plasma chemistry of the ploughshare tortoise (Astrochelys yniphora) in a captive breeding program. Journal of Zoo and Wildlife Medicine, 48, $102-115$.

Martin A (1998) Captive breeding and maintenance of the royal python (Python regius). British Herpetological Society Bulletin, (64), 15-20.

Martin RO, Senni C, D’Cruze NC (2018) Trade in wild-sourced African grey parrots: Insights via social media. Global Ecology and Conservation, 15 , e00429.

Platt SG, Monyrath V, Sovannara H, Kheng L, Rainwater TR (2012) Nesting phenology and clutch characteristics of captive Siamese crocodiles (Crocodylus siamensis) in Cambodia. Zoo Biology, 31, 534-545.

Robinzon B, Nir I, Lapid R (2005) Growth and body composition in captive Testudo graeca terrestris fed with a high-energy diet. Applied Herpetology, 2, 201-209. Stearns BC (2007) The captive status of the African spurred tortoise Geochelone sulcata: Recent developments. International Zoo Yearbook, $28,87-98$.

Stephen C, Pasachnik S, Reuter A, Mosig P, Ruyle L, Fitzgerald L (2011) Survey of status, trade, and exploitation of Central American iguanas. Report, Department of Interior, United States Fish and Wildlife Service, Washington, DC, USA.

Uhrig D, Lee DS (2006) Care and captive breeding of the highly endangered Vietnam pond turtle, Mauremys annamensis (Siebenrock, 1903). Radiata, 151(1), 3-10. Vyas R (2001) Breeding of the Indian roofed turtle Kachuga tecta in captivity. Zoos' Print Journal, 16, 600-603. 
Vyas R (2005) Captive breeding of the Indian star tortoise (Geochelone elegans). Zoos' Print Journal, 20, $1859-1864$.

Yang JD (2016) Artificial breeding method of Chelydra serpentina osceola. China, CN103843723B. (in Chinese) [杨君达 (2016) 一种鳄龟人工繁殖方法. 中国， CN103843723B.] 